\title{
MOTT TRANSITTON OF THE ELECTRON-HOLE LIQUID IN Ge
}

Leonard M. Sander and D. K Fairobent

Physics Department, University of Michigan Ann Arbor, Michigan 48109

(Received 5 November 1975; in revised form 5 August 1976 by H. Suhl)

A treatment is given of the Mott transition of a gas of excitons in equilibrium with electron-hole droplets in $\mathrm{Ge}$. We find that transition occurs near $4 \mathrm{~K}$. We can come to no firm conclusion whether the Mott transition is a first-order transition separate from the liquid-gas transition, as has been suggested.

In recent years there has been considerable interest in the properties of the electron-hole plasma in optically excited $\mathrm{Ge} .1,2,3$ It is known that the plasma condenses into liquid droplets when the density is sufficiently high and the temperature is sufficiently low. The gas which is in equilibrium with the liquid is throught to be a mixture of excitons, whereas at sufficiently high densities the gas should be completely ionized. In this paper we propose to give an approximate theoretical description of the transition between the ionized plasma and the exciton gas. This transition is similar in nature to the transition from metallic to insulating behavior in solids which was first described by Mott. ${ }^{4}$

A good deal is now known about the phase diagram of the electron-hole system, both experimentally ${ }^{5}, 8$ and theoretically ${ }^{7-12}$. For example, the gas-liquid critical temperature $(T=6.5 \mathrm{~K})$ and density $\left(\mathrm{n}_{\mathrm{c}} \approx 8 \times 10^{16} \mathrm{~cm}^{-3}\right)$ have been measured ${ }^{5}$ and several calculations have appeared ${ }^{7-10}$.

We now consider a calculation of the phase diagram of the system consisting of a mixture of an electron-hole plasma and a gas of excitons. We treat the excitons as composite particles, i.e., as a species distinct from the electrons and holes. For each temperature, the chemical potential of the system is calculated as a function of the total density of particle pairs $n_{\text {tot }}=n_{p}+n_{e x}$ ( $n_{p}=$ density of particle pairs in the plasma, $\mathrm{n}_{\text {ex }}=$ density of excitons). The

phase diagram then follows from a Maxwell construction.

In our model for the plasma in Ge the conduction band has four equivalent valleys, with longitudinal mass $\mathrm{m}_{\mathrm{el}}=1.58 \mathrm{~m}$, and transverse mass $\mathrm{m}_{\mathrm{et}}=0.082 \mathrm{~m}$ ( $\mathrm{m}$ is the bare electron mass). There are two hole bands with masses $m_{H}=0.347 \mathrm{~m}$ and $m_{L}=0.042 \mathrm{~m}$; the exciton mass is

$$
m_{e x}=\left(m_{e I}+\frac{m_{H} m_{L}}{m_{H}+m_{L}}\right)^{1 / 3}\left(m_{e t}+\frac{m_{H} m_{L}}{m_{H}+m_{L}}\right)^{2 / 3} .
$$

It is convenient to measure lengths in units of the exciton Bohr radius. Energy is measured in units of twice the exciton Rydberg and mass in units of the optical reduced mass.
We write the free energy per unit volume as:

$$
f_{\text {tot }}=f_{p}\left(n_{p}, T\right)+f_{e x}\left(n_{e x}, n_{\text {tot }}, T\right)
$$

where $f_{p}$ and $f_{\text {ex }}$ refer to the plasma and excitons, respectively. For the plasma:

$$
\begin{gathered}
f_{p}\left(n_{p}, T\right)=f_{e}^{o}\left(n_{p}, T\right)+f_{h}{ }^{\circ}\left(n_{p}, T\right)+ \\
f^{\operatorname{exch}}\left(n_{p}\right)+f^{\operatorname{corr}}\left(n_{p}\right) .
\end{gathered}
$$

Here, $f_{e}{ }^{\circ}\left(n_{p}, T\right)$ is the free energy of a noninteracting gas of electrons, $f_{h}{ }^{\circ}\left(n_{p}, T\right)$ is the free energy of a non-interacting gas of holes, $f^{\text {exch }}\left(n_{p}\right)$ is the exchange energy of the plasma, $f^{\operatorname{corr}}\left(n_{p}\right)$ is the correlation energy of the plasma.

The quantities $f_{e}{ }^{\circ}\left(n_{p}, T\right)$ and $f_{h}{ }^{\circ}\left(n_{p}, T\right)$ are calculated by numerical integration of standard formulae of classical statistical mechanics. The exchange and correlation energy are taken to be independent ${ }^{5}$ of $T$. For the exchange energy we use the zero temperature result of Vashishta, et.al. ${ }^{13}$

$$
f^{\text {exch }}\left(n_{p}\right)=-0.92714 n_{p}^{4 / 3} .
$$

For the correlation energy, we use the tabulated results of Vashishta, et.al., ${ }^{13}$ for $n>0.03$, and a Wigner-type formula,

$$
f^{\operatorname{corr}}\left(n_{p}\right)=\frac{A}{B+n_{p} 1 / 3} n_{p}^{4 / 3}
$$

for $\mathrm{n}<0.03$. A and $B$ are chosen to match smoothly with the tabulated results at $n=0.03$. The free energy of the excitons is

$$
\begin{aligned}
& f_{e x}\left(n_{e x}, n_{t o t}, T\right)=f_{e x}{ }^{o}\left(n_{e x}, T\right)+ \\
& f_{e x}{ }^{q}\left(n_{e x}, T\right)+n_{e x} E_{B}\left(n_{t o t}, T\right) .
\end{aligned}
$$

Here $f_{e}^{\circ}\left(n_{e x}, T\right)$ is the free energy of a classical van der Waals gas of particle of mass $m_{e x}$, and $f_{e x}{ }^{q}\left(n_{e x}, T\right)$ is the first quantum correction given by 


$$
\mathrm{f}_{\mathrm{ex}} \mathrm{q}\left(\mathrm{n}_{\mathrm{ex}}, \mathrm{T}\right)=\frac{\pi^{3 / 2}}{32} \frac{\mathrm{n}_{\mathrm{ex}}^{2}}{(\mathrm{kT})^{1 / \mathrm{L}_{\mathrm{m}}} \mathrm{m}_{\mathrm{ex}}^{3 / 2}}
$$

$E_{B}\left(n_{\text {tot }}, T\right)$ is the binding energy of the exciton taking into account the effects of screening as discussed below. All interaction effects other than the effect of the plasma on the binding energy of the excitons are neglected.

For a system of fixed total density $n_{\text {tot }}$ at

$$
f_{\text {tot }}\left(n_{e x}, n_{p}\right)=f_{p}+f_{e x}
$$

temperature $T$ we minimize

with respect to $x$, where $x=n_{p} / n_{\text {tot }}$. In this way, we find the free energy of the system and the values of $n_{p}$ and $n_{e x}$ as functions of $n_{\text {tot }}$. The chemical potential of the system is then given by

$$
\begin{aligned}
& \mu_{\text {tot }}=\partial f_{\text {tot }} / \partial n_{\text {tot }}=\mu_{p} \partial n_{p} / \partial n_{\text {tot }}+\mu_{e x} \partial n_{e x} / \partial n_{t o t} \\
& +\partial_{e x} / \partial n_{\text {tot }} E_{B}+n_{e x} \partial E_{B} / \omega_{\text {tot }} \text {, }
\end{aligned}
$$

where $\mu_{p}$ and $\mu_{e x}$ are derivatives of $f_{p}$ and $f_{e x}$ with respect to $n_{p}$ and $n_{e x}$, respectively. Now using the fact that $n_{e x}{ }^{+} n_{p}=n_{t o t}$, we can write (9) in two equivalent forms:

$$
\begin{gathered}
\mu_{\text {tot }}=\mu_{p}+n_{\text {ex }} \partial E_{B} / \partial n_{\text {tot }}, \\
\mu_{\text {tot }}=\mu_{e x}+E_{B}+n_{e x} \partial E_{B} / \partial n_{\text {tot }} .
\end{gathered}
$$

The central physical quantity in our calculation is $\mathrm{E}_{\mathrm{B}}$. As we will see the nature of the Mott transition depends very sensitively on $\mathrm{E}_{\mathrm{B}}$, which is, unfortunately, hard to calculate.

Note that we cannot take $\mathrm{E}_{\mathrm{B}}$ to be independent of $n_{\text {tot }}$ as a simple application of the Mott criterion shows. ${ }^{4}$

We now discuss estimates for $E_{B}$. To this

end we introduce an auxiliary quantity, $\epsilon$, which is the binding energy of an exciton relative to its own continurm, i.e., relative to the (renormalized) band edge. Note that this is not the same as $E_{B}$, which is measured with respect to the band edge at $n_{p}=0$. If we consider the exchange and correlation to give rise to a "rigid" shift of the bands" ${ }^{5}$, we can write:

$$
E_{B}=\epsilon+\mu_{\mathrm{Xc}},
$$

where $\mu_{x c}$ is the exchange and correlation part of $\mu_{p}$. It has been shown ${ }^{11}$ that for small $n_{p}$ (in the Debye-Huckel limit) the shifts in $\epsilon$ and $\mu_{x c}$ cancel, leaving $E_{B}$ independent of $n_{p}$. Our theory does not have the correct limiting behavior: because of the assumptions implicit in Eq. (2) we do not attain the Debye-Huckel limit. Nevertheless the renormalization is partially included in (12), and the errors may not be serious for a global picture of the phase diagram.

At low densities (and low temperatures) where the gas consists entirely of excitons one can use the results of collision theory to find $\Delta \epsilon$. This shift is given by ${ }^{14}$

$$
\Delta \varepsilon=-\frac{\text { an }}{m_{\text {ex }}}-100 \mathrm{n}^{4 / 3}
$$

where $a$ and $\alpha$ are, respectively, the scattering length and polarizability of the exciton. If there are few excitons, one can find the binding energy by computing the eigenvalues of the Bethe-salpeter equation ${ }^{2}$ which takes into account the effects of the plasma:

$$
\begin{gathered}
\epsilon \varphi(k)=\frac{1}{2 m} \cdot k^{2} \varphi(k)+\int \frac{d k^{\prime}}{(2 \pi)^{3}} v\left(k-k^{\prime}\right) \\
\varphi\left(k^{\prime}\right)\left(1-f_{e}\left(k^{\prime}\right)-f_{h}\left(k^{\prime}\right)\right) .
\end{gathered}
$$

Here $\mathrm{v}(\mathrm{k})$ is the Fourier transform of the screened Coulomb potential, $f(k)$ is the Fermi distribution function, $m$ is the reduced mass, and $\varphi(k)$ is the center of mass wavefunction.

The potential $\mathrm{v}(\mathrm{k})$ is given by

$$
v(k)=4 \pi e^{2} /\left(k^{2}+\lambda^{2}\right)
$$

and $\lambda$, the inverse screening length, is given by standard (temperature dependent) formulas for screening by a gas of free electrons and free holes. We solve $\mathrm{Eq}$. (14) variationally. The solution of the B-S equation for $\mathrm{T}=2^{\circ}$ is shown in Figure 1.

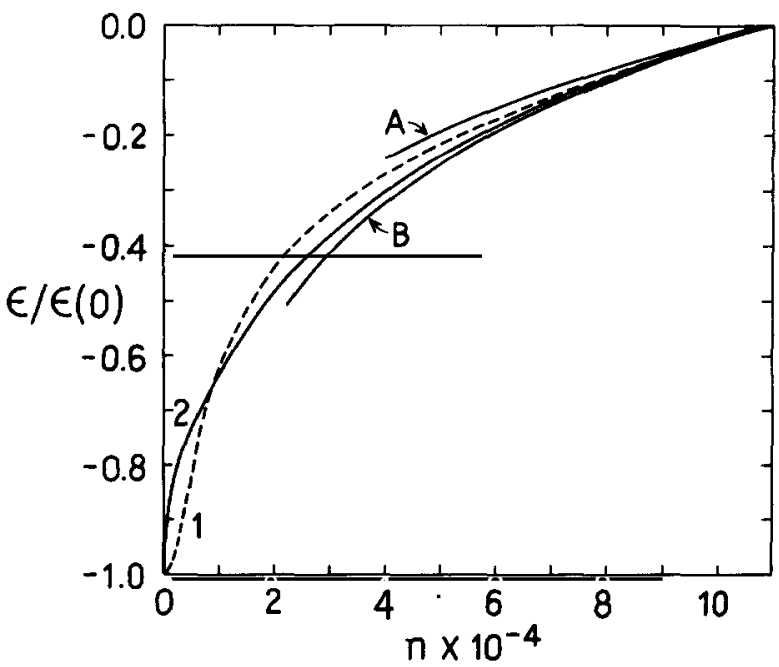

Figure 1 The exciton binding energies $\epsilon$ as a function of density for $T=2^{\circ}$. The density is in dimensionless units and $\boldsymbol{\varepsilon}(\mathrm{n}=0)=4.15 \mathrm{mev}(\operatorname{Ref} .16)$. Curve $\mathrm{A}$ is the result of a Bethe-Salpeter calculation, and curve $B$ are the scaled results of Ref. 15. For interpolations 1 and 2, see text. 
Another way to find $\epsilon$ at high densities is to make use of the measured values of the ionization energy of donor states in Ge. Compton and Davis ${ }^{15}$ have measured the ionization energy of donor states in antimony-doped Ge for $6.6 \times 10^{15} \mathrm{~cm}^{-3}<n_{\text {donors }}<2 \times 10^{17} \mathrm{~cm}^{-3}$. The donor state is analogous to the exciton state and by scaling the measured results in appropriate dimensionless units (which tokes into account the different screening and different masses in our case) one can estimate the exciton binding energy. The results obtained in this way (the curve labeled B in Figure 1) are quite similar to the solution of the B-S equation at high densities.

One can interpolate between the low density and high density limits to obtain $\epsilon$ for a.ll densities. Unfortunately, since we have no reliable values at intermediate densities, the choice of an interpolating function is somewhat arbitrary. We have calculated the phase diagram for several choices of interpolating functions. The curves labeled 1 and 2 in Figure 1 are two representative choices of $\epsilon$, and the associated $\mathrm{E}_{\mathrm{B}}$ are shown in Figure 2 .

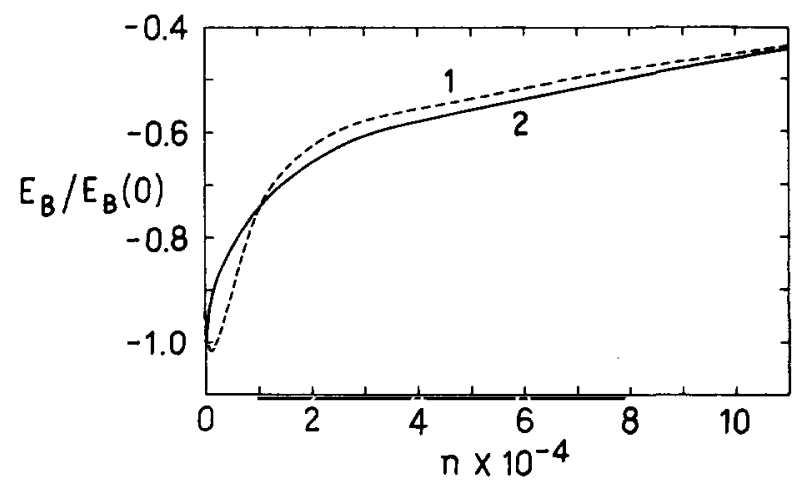

Figure 2 The exciton binding energy $E_{B}$ as a function of density. The units are the same as in Figure 1. For interpolations 1 and 2 , see text.

For the gas-liquid transition we find $\mathrm{T}_{\mathrm{c}}=7.5^{\circ}$ and $\mathrm{n}_{\mathrm{c}}=4 \times 10^{16} \mathrm{~cm}^{-3}$. This is independent of the choice of exciton binding energy since the gas is completely ionized in this region.

The shape of the phase diagram at densities much less than $n$ depends on the particular choice of the exciton binding energy. The curve labeled No. 1 in Figure 3 is the phase diagram which we obtain when we use the binding energy curve No.I. In this case the Mott transition appears as a first order phase transition which is separate from the gas-liquid transition, with a critical temperature of $8.5^{\circ}$ and a critical density of $10^{14} \mathrm{~cm}^{-3}$. The concentration of excitons along the co-existence curve is plotted as a function of $T$ in Figure 4. With binding energy No. 2 we obtain the phase diagram label No. 2 in Figure 3. In this case the Mott transition does not appear as a first order phase transition separate from the gas- liquid transition, but as a shoulder on the curve.

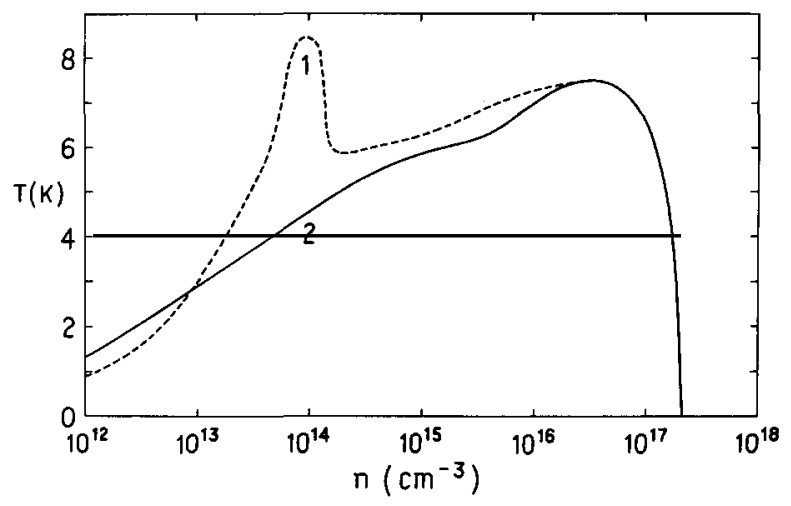

Figure 3 Phase diagrams corresponding to the two choices of exciton binding energy shown in Figure 1 .

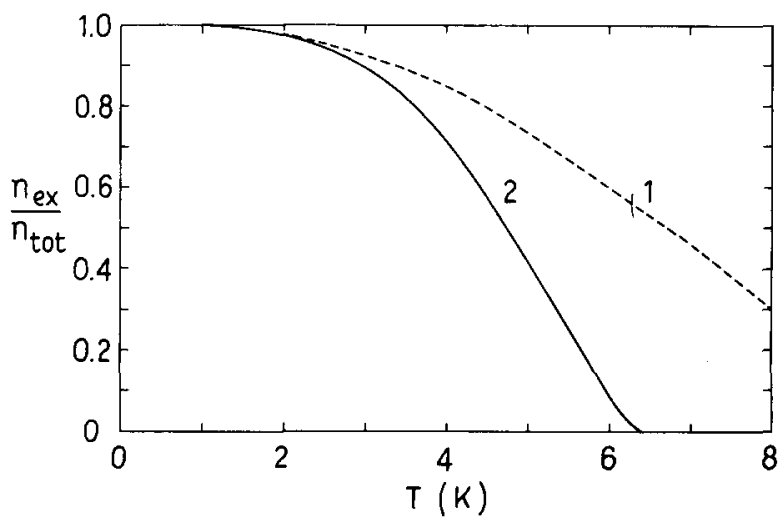

Figure 4 The concentration of excitons along the co-existence curve as a function of temperature. The curve labeled 1 refers to the portion of the phase diagram on the far left in Fig. 2. The other branches have a very small exciton concentration.

Our results should be compared to those of ref. 11 and 12 where the classical limit is very carefully investigated. Unfortunately, these authors adopt a rather arbitrary interpolation for the function corresponding to our $\epsilon$, and obtain only a phase diagram corresponding to case 1. The major point of our calculation is the extreme sensitivity of the phase diagram to $\epsilon$ or $E_{B}$. We believe that in view of the complexity of the theoretical probelm no firm conclusion can be drawn about the nature of $E_{B}$ for intermediate densities.

We feel that the question of the existence of a first order transition in this system will have to be settled by experiment. To our knowledge the only relevant experiment to date is that of Thomas, et.al., 17 who detect what may be the 
beginning of a "shoulder" of type 2. Thus the question remains open.

Acknowledgement-We would like to acknowledge helpful conversations and communications with V. K. Wong, H. B. Shore, and G. A. Thomas.

\section{REFERENCES}

* Supported by the National Science Foundation.

1. POKROVSKII, YA. E., Phys. Stat. Sol. (a) 11, 385 (1972).

2. BRINKMAN, W. F. and RICE, T. M., Phys. Rev. B 7, 1508 (1973).

3. COMBESCOT, M. and NOZIERES, P., J. Phys. C: Solid State Phys. $\underline{5}, 2369$ (1972).

4. MOTT, N. F., Rev. Mod. Phys. 40, 677 (1968).

5. THOMAS, G. A.,RICE, R. M. and HENSEL, J. C., Phys. Rev. Letters 33, 219 (1974).

6. IO, T. K., FELDMAN, B. J. and JEFFRIES, C. D. Phys. Rev. Letters 31, 224 (1973).

7. COMBESCOT, M.; Phys. Rev. Letters 32, 15 (1974).

8. VASHISHTA, P., DAS SHASHIKALA, G., and SINGWI, K. S., Phys. Rev. Letters 33, 911 (1974).

9. REINECKE, T. L. and YING, S. C., Phys. Rev. Letters 35, 311 (1975).

10. DROZ, M. and COMBESCOT, M., Phys. Letters 51A, $473\left(\frac{37}{1975)}\right.$.

11. KREMP, D., EBELING, W. and KRAEFT, W. D., Phys. Stat. Sol. (b) 69, K59 (1975).

12. KRAEFT, W. D., KILLIMAN, K. and KREMP, D., Phys. Stat. Sol. (b) 72, 461 (1975).

13. BHATTACHARYYA, P., MASSIDA, V., SINGWI, K. S. and VASHISHTA, P., Phys. Rev. B 10, 5127 (1974).

14. MASSEY, H. S. W. and BURHOP, E. H. S., Electronic and Ionic Impact Phenomena p. 178. Oxford (1952).

15. DAVIS, E. A. and COMPTON, W. D., Phys. Rev. 140, 2183 (1965).

16. FROVA, A., THOMAS, G. A., MLLLER, R. E. and KANE, E. O., Phys. Rev. Letters 34,1572 (1975).

17. THOMAS, G. A., FROVA, A., HENSEL, J. C., MILLER, R. E. and LEE, P. A., Phys. Rev. B13, 1692 $(1976)$. 